\title{
Radiation excited by a charged-particle bunch on a planar periodic wire structure
}

\author{
Andrey V. Tyukhtin," Viktor V. Vorobev, ${ }^{\dagger}$ and Sergey N. Galyamin ${ }^{\ddagger}$ \\ Physical Faculty of St. Petersburg State University, St. Petersburg 198504, Russia
}

(Received 24 May 2014; published 18 December 2014)

\begin{abstract}
The electromagnetic field of a bunch moving in the presence of a plane grid composed of thin parallel wires is considered by using the averaged boundary conditions method. Two different cases of motion are examined. In the first one, the bunch moves at a constant distance from the grid orthogonally to the wires. The excited surface wave is presented in the form of a spectral integral for a thin bunch with an arbitrary longitudinal profile. The wave propagates along the wires and does not decay with distance (if dissipation is negligible). Energy losses of the bunch over a unit path are obtained. In the second case, the bunch orthogonally crosses the wire grid. The volume and surface waves are separately analyzed. Properties of the spectral angular density of energy of volume radiation in the far-field zone are described. The energy losses due to the volume and surface radiation are determined. It is demonstrated that the structure of the surface waves in both cases allows determination of the length of the bunch.
\end{abstract}

DOI: 10.1103/PhysRevSTAB.17.122802

PACS numbers: 41.60.Dk, 41.75.-i

\section{INTRODUCTION}

The investigation of charged-particle radiation in the presence of periodic structures with small spacing is interesting for the diagnostics of bunches, generation of radiation, and screening of electromagnetic fields. Recently, the radiation of bunches in volume (3D) structures that consist of parallel wires was considered [1-4] (such a structure is frequently called a "wire metamaterial"). In particular, it has been shown that a charge moving orthogonally to wires generates nondivergent radiation that concentrates near certain lines behind the charge. Because of these properties, such structures can be used to determine the bunch sizes.

However, 3D wire structures can be difficult to use in practice. Therefore, it is reasonable to consider planar (2D) structures composed of parallel wires. Although such problems were studied in a series of papers [5-9], the authors analyzed only the case of a point charge and mainly focused on a volume part of the radiation. Here, we study the radiation of charged-particle bunches with an arbitrary longitudinal profile and consider both volume radiation and surface radiation. As we further show, the surface wave is convenient to measure the bunch size. Note that we consider the "nonideality" of the structure using the averaged boundary conditions [10], which allow us to take

\footnotetext{
*tyukhtin@bk.ru

vorobjovvictor@gmail.com

galiaminsn@yandex.ru
}

Published by the American Physical Society under the terms of the Creative Commons Attribution 3.0 License. Further distribution of this work must maintain attribution to the author(s) and the published article's title, journal citation, and DOI. into account both the geometry parameters and the finite conductivity of the wires.

\section{MOVEMENT ALONG THE GRID}

We consider a periodical set of infinitely long cylindrical conductors in the plane $z=0$ and parallel to the $x$ axis (Fig. 1). The diameter of each wire is $2 r_{0}$, and the period of the structure is $a$. A charged-particle bunch moves along the grid perpendicularly to the wires with constant velocity $\vec{v}=v \overrightarrow{\mathbf{e}}_{y}, v=c \beta$. The distance between the grid and the trajectory of motion is $a_{0}$ [Fig. 2(a)]. This bunch has infinitesimal size in the transverse directions (along the $x$ and $z$ axes) and some longitudinal distribution of charges $\eta(\xi)$, where $\xi \equiv y-v t$. The charge density $\rho$ and the current density $\vec{j}$ are given by the following expression:

$$
\rho=\delta(x) \delta\left(z+a_{0}\right) \eta(\xi), \quad \vec{j}=v \rho \overrightarrow{\mathbf{e}}_{y},
$$

where $\delta(x)$ is the Dirac delta function.

We present the total field as the sum of an "incident" field (which is a field of the bunch in infinite vacuum without borders) and some additional field that is induced by the presence of the grid. The incident field can be described by using a single-component vector potential $\vec{A}^{i}$ and an associated scalar potential $\Phi^{i}$, which can be written as the Fourier integrals:

$$
\begin{aligned}
\vec{A}^{i} & =A_{y}^{i} \overrightarrow{\mathbf{e}}_{y}, \quad \Phi^{i}=\beta^{-1} A_{y}^{i}, \\
A_{y}^{i} & =\int_{-\infty}^{\infty} A_{y \omega}^{i} e^{-i \omega t} d \omega, \\
A_{y \omega}^{i} & =\frac{i}{c} \int_{-\infty}^{\infty} d k_{x} \frac{\tilde{\eta}(\omega / v) e^{i k_{x} x+i k_{z 0}\left|z+a_{0}\right|+i \omega y / v}}{k_{z 0}},
\end{aligned}
$$




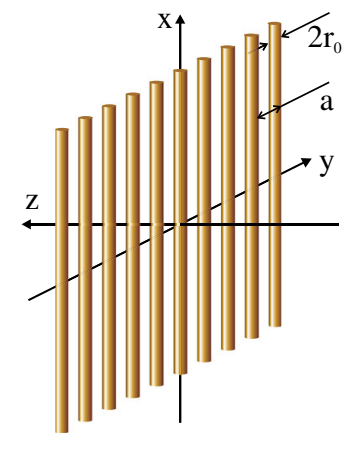

FIG. 1. Planar wire grid.

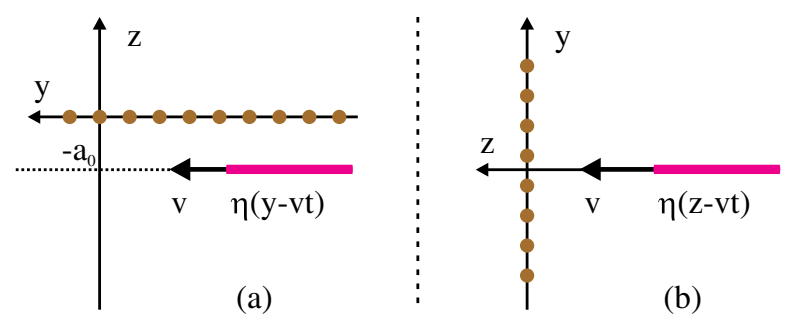

FIG. 2. Schemes of bunch movement.

where $\tilde{\eta}(k)$ is the Fourier transform of the bunch profile

$$
\tilde{\eta}(k)=\frac{1}{2 \pi} \int_{-\infty}^{\infty} \eta(\xi) \exp \{-i k \xi\} d \xi
$$

$k_{z 0}=\sqrt{-\frac{\omega^{2}}{c^{2}} \frac{1-\beta^{2}}{\beta^{2}}-k_{x}^{2}}$, and the root is fixed so that

$$
\begin{aligned}
& \operatorname{sgn}(\sqrt{s})=\operatorname{sgn}(\omega), \quad \text { if } \operatorname{Im}(\sqrt{s})=0, \\
& \operatorname{Im}(\sqrt{s})>0, \quad \text { if } \operatorname{Re}(\sqrt{s})=0 .
\end{aligned}
$$

We assume that the wavelength under consideration and the scale of the spatial variation of the incident field are greater than the grid's period, which is much greater than the wires' thickness:

$$
a \ll c \beta /\left(\omega \sqrt{1-\beta^{2}}\right), \quad a \ll 2 \pi c / \omega, \quad r_{0} \ll a .
$$

In this situation, the method of the averaged boundary conditions (ABCs) can be used [10]. This technique is actively applied for analysis of the influence of various grid structures on electromagnetic fields. In contradiction to other methods, it allows one easily to take into account a series of grid structure features, such as a form of a grid cell, a form of wires cross section, and limited conductivity of wires. These conditions describe the averaged field over the lattice period. For a structure of parallel conductors, the $\mathrm{ABCs}$ can be written in the following form [10]:

$$
\begin{gathered}
\left.E_{x \omega}\right|_{z=0}=-\frac{c}{4 \pi}\left(A+B \frac{\partial^{2}}{\partial x^{2}}\right)\left\{H_{y \omega}\right\}, \\
\left\{E_{x \omega}\right\}=\left\{E_{y \omega}\right\}=\left\{H_{x \omega}\right\}=0,
\end{gathered}
$$

where \{\} denotes the jump of the corresponding value at $z=0$, i.e., $\{f(z)\}=f(+0)-f(-0)$,

$$
A=a\left[Z-2 i \frac{\omega}{c^{2}} \ln \left(\frac{a}{2 \pi r_{0}}\right)\right], B=-2 i \frac{a}{\omega} \ln \left(\frac{a}{2 \pi r_{0}}\right) .
$$

Here, $Z$ is a wire impedance, which has a simple approximate representation for two particular cases [10]:

$$
Z \approx \frac{1}{\sigma_{e}} \begin{cases}\left(\pi r_{0}^{2}\right)^{-1}, & r_{0} \ll 2 d, \\ \left(\sqrt{2} \pi r_{0} d\right)^{-1} \exp \{-i \pi / 4\}, & r_{0} \gg 2 d,\end{cases}
$$

where $\sigma_{e}$ and $d$ are the conductivity and the thickness of the skin layer for the wire material, respectively.

The additional field, which is induced by the grid presence, can also be described by using the singlecomponent vector potential. In order to describe this part of the field, it is convenient to use the component parallel to the wires: $\vec{A}^{b}=A_{x}^{b} \overrightarrow{\mathbf{e}}_{x}$. Using the $\mathrm{ABC}$ (6), after some transformations, one can obtain the following result:

$$
\begin{aligned}
A_{x \omega}^{b}= & \frac{i \omega}{c^{2} \beta} \int_{-\infty}^{\infty} d k_{x} \tilde{\eta}(\omega / v) \\
& \times \frac{k_{x} \exp \left\{i k_{x} x+i \frac{\omega}{v} y+i k_{z 0}\left(|z|+a_{0}\right)\right\}}{k_{z 0}\left[\left(\frac{\omega^{2}}{c^{2}}-k_{x}^{2}\right)\left(1-i \tilde{\kappa} k_{z 0}\right)+\delta k_{z 0} \frac{\omega}{c}\right]},
\end{aligned}
$$

where

$$
\tilde{\kappa}=\kappa c / \omega=\frac{a}{\pi} \ln \frac{a}{2 \pi r_{0}}, \quad \delta=\frac{a c Z}{2 \pi} .
$$

One can observe that the integrand in expression (9) has poles, which determine a surface wave. For perfect wires $(\delta=0)$, the poles are at $k_{x}= \pm \omega / c$ and give the contribution, which describes the surface wave

$$
\begin{aligned}
A_{x \omega}^{s}= & -\operatorname{sgn}(x \omega) \frac{i \pi}{c} \tilde{\eta}(\omega / v) \\
& \times \frac{\exp \left\{i \frac{\omega}{c}|x|+i \frac{\omega}{v} y-\frac{|\omega|}{v}\left(|z|+a_{0}\right)\right\}}{1+\tilde{\kappa} \frac{|\omega|}{v}} .
\end{aligned}
$$

One can show that the contributions of other singularities in integral (9) are either zero or exponentially small everywhere in the far-field zone $\omega / c \cdot R \gg 1$. This means that the Smith-Purcell radiation cannot be taken into account within the considered "long-wave" approximation. The components of the electromagnetic field of the surface wave are 


$$
\begin{gathered}
E_{x \omega}^{s}=H_{x \omega}^{s}=0 \\
\left\{\begin{array}{c}
E_{y \omega}^{s} \\
E_{z \omega}^{s}
\end{array}\right\}= \\
\operatorname{sgn} x\left\{\begin{array}{c}
H_{z \omega}^{s} \\
-H_{y \omega}^{s}
\end{array}\right\}=\frac{-\pi \omega}{c^{2} \beta}\left\{\begin{array}{c}
\operatorname{sgn} \omega \\
i \operatorname{sgn} z
\end{array}\right\} \tilde{\eta}(\omega / v) \\
\times \frac{\exp \left\{i \frac{\omega}{c}|x|+i \frac{\omega}{v} y-\frac{|\omega|}{v}\left(|z|+a_{0}\right)\right\}}{1+\tilde{\kappa} \frac{|\omega|}{v}} .
\end{gathered}
$$

Expressions (13) allow one to calculate the surface wave of a bunch with arbitrary longitudinal profile (3). If the profile is symmetrical, then the Fourier transform (3) is even and real, and one can obtain the field of the surface wave in the form of the integral over a positive semiaxis:

$$
\left\{\begin{array}{l}
E_{y}^{s} \\
E_{z}^{s}
\end{array}\right\}=2 \pi \beta \int_{0}^{\infty} d k^{\prime}\left\{\begin{array}{c}
-\cos \left(k^{\prime} \hat{y}\right) \\
\operatorname{sgn} z \sin \left(k^{\prime} \hat{y}\right)
\end{array}\right\} \frac{k^{\prime} \tilde{\eta}\left(k^{\prime}\right) e^{-k^{\prime} z}}{1+\tilde{\kappa} k^{\prime}},
$$

where $k^{\prime}=\omega / v$,

$$
\hat{y}=y+\beta(|x|-c t), \quad \hat{z}=|z|+a_{0} .
$$

The examples of such symmetrical "filaments" are the bunches with rectangular and Gaussian charge distributions:

$$
\begin{aligned}
\eta^{\text {rect }}(\xi) & =\frac{q}{2 \sigma} \Theta(\sigma-|\xi|), \\
\tilde{\eta}^{\text {rect }}(k) & =\frac{q}{2 \pi} \frac{\sin (k \sigma)}{k \sigma}, \\
\eta^{\text {Gaus }}(\xi) & =\frac{q}{\sigma \sqrt{2 \pi}} \exp \left\{-\frac{\xi^{2}}{2 \sigma^{2}}\right\}, \\
\tilde{\eta}^{\text {Gaus }}(k) & =\frac{q}{2 \pi} \exp \left\{-\frac{k^{2} \sigma^{2}}{2}\right\},
\end{aligned}
$$

or the particular case of a point charge

$$
\eta^{\mathrm{pnt}}(\xi)=q \delta(\xi), \quad \tilde{\eta}^{\mathrm{pnt}}(k)=\frac{q}{2 \pi}
$$

The exact expression in terms of elementary functions can be obtained for a perfectly conducting plane in the $x$ direction $(a \rightarrow 0, \tilde{\kappa} \rightarrow 0)$, when considering the rectangular bunch [11]:

$$
\left\{\begin{array}{c}
E_{y}^{s} \\
E_{z}^{s}
\end{array}\right\}_{\text {rect }}=q \beta \frac{\left\{\begin{array}{c}
\hat{y}^{2}-\hat{z}^{2}-\sigma^{2} \\
2 \hat{y} \hat{z} \operatorname{sgn} z
\end{array}\right\}}{\left[\hat{z}^{2}+(\hat{y}+\sigma)^{2}\right]\left[\hat{z}^{2}+(\hat{y}-\sigma)^{2}\right]} .
$$

Corresponding values for the Gaussian bunch can be expressed through the complementary error function [11].

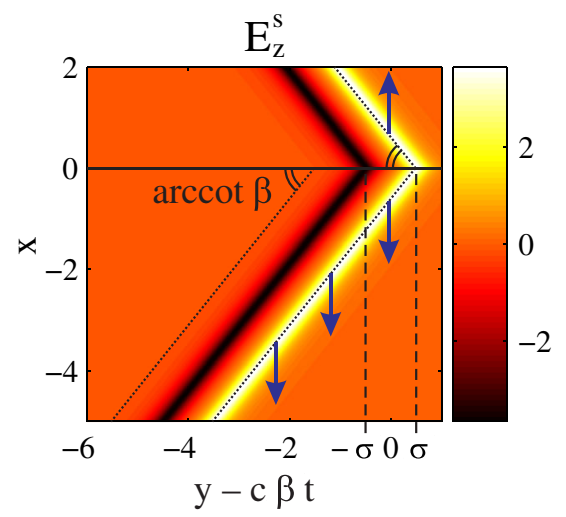

FIG. 3. The structure of the $E_{z}$ component of the surface wave in the plane of the grid for the "rectangular" bunch with $q=1$ esu. Gaussian units are used. The bunch length is $2 \sigma=1 \mathrm{~cm} ; \tilde{\kappa}=0, \delta=0$ (the grid is a perfectly conducting plane in the $x$ direction); $a_{0}=0.2 \mathrm{~cm} ; \beta=0.8$, and $z=+0$. The solid blue arrows show the direction of the wave's energy flow. Dashed lines are the straights parallel to $y=-\beta(|x|-c t)$.

The surface wave propagates along the wires at the speed of light in vacuum. Its shape does not change with time, and the wave does not decrease (if $\delta=0$ ) when the observation point is shifted along the line $y=-\beta(|x|-c t)$ (Fig. 3). The numerical results for the rectangular bunch are presented in Fig. 4, where both the field components and the energy flow density

$$
\begin{aligned}
\vec{S}^{s} & =S^{s} \operatorname{sgn}(x) \overrightarrow{\mathbf{e}}_{x}=\frac{c}{4 \pi}\left|\left[\vec{E}^{s}, \vec{H}^{s}\right]\right| \operatorname{sgn}(x) \overrightarrow{\mathbf{e}}_{x} \\
& =\frac{c}{4 \pi}\left[\left(E_{y}^{s}\right)^{2}+\left(E_{z}^{s}\right)^{2}\right] \operatorname{sgn}(x) \overrightarrow{\mathbf{e}}_{x}
\end{aligned}
$$

are shown. The pictures represent spatial distribution of the electric field and the energy flow of the surface wave in the plane orthogonal to the wires $(x=$ const $>0$ ) (see Fig. 5 for reference). The distance between extrema of the field components corresponds to the length of the bunch. These images do not change with time if dissipation is negligible. Thus, the surface wave can be used to estimate the length of the bunch. But if the distance between the charge and the grid is of the same order as the length of the bunch, then these extrema become undistinguishable that complicates bunch size determination.

Note that the surface wave under consideration and the radiation of the bunch in the wire metamaterial have similar structures [1,4]. However, the planar structure is easier to implement in the accelerator technique.

The surface wave is the only source of charge losses in the case under consideration. We use the energy losses of the bunch over a unit path to describe energy characteristics of the surface wave. The total energy passing through a unit square over the entire time can be calculated as $\int_{-\infty}^{\infty} S_{x} d t$. The total energy transported by the surface wave is obtained by integrating over planes $x= \pm x_{0}$ $\left(x_{0}=\right.$ const $\left.>0\right)$ : 

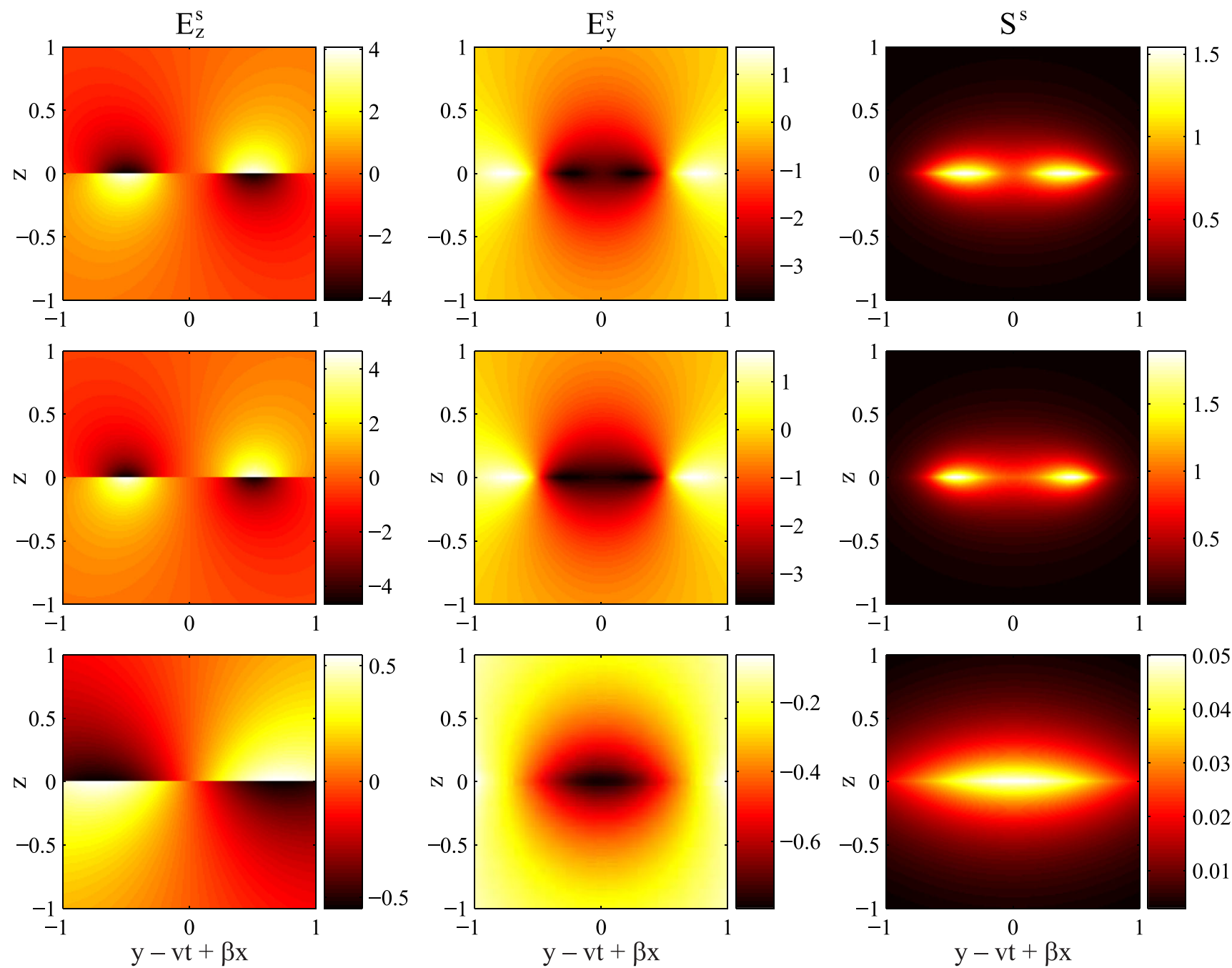

FIG. 4. The field components and the energy flow density of the surface wave from the rectangular bunch with $q=1$ esu moving along the wire grid. Gaussian units are used. The bunch length is $2 \sigma=1 \mathrm{~cm}$ for all pictures; $\tilde{\kappa}=0.0148 \mathrm{~cm}(a=0.1 \mathrm{~cm}$, $\left.r_{0}=0.01 \mathrm{~cm}\right), a_{0}=0.2 \mathrm{~cm}$ for the first row, $\tilde{\kappa}=0, a_{0}=0.2 \mathrm{~cm}$ for the second row, $\tilde{\kappa}=0, a_{0}=1 \mathrm{~cm}$ for the third row; $\delta=0$, $x=$ const $>0$, and $\beta=1$.
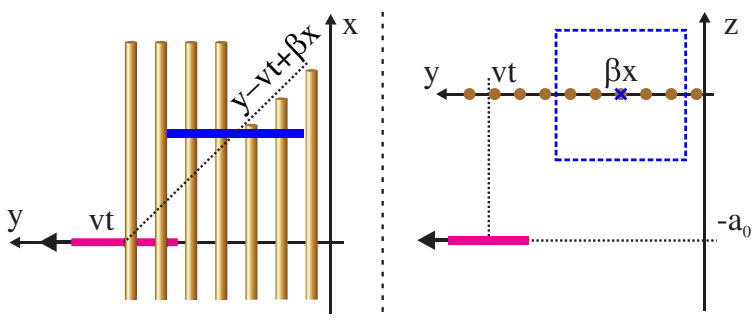

FIG. 5. Relative position of "snapshots" area, bunch, and wires for surface wave field distributions in Fig. 4. The area with calculated distributions is shown as a solid blue horizontal line on the left projection and as a square with a dashed blue border on the right projection. The bold arrow shows the direction of the bunch motion.

$$
W^{s}=\iint_{x= \pm x_{0}} d y d z \int_{-\infty}^{\infty} S_{x} d t .
$$

After some transformations, using expressions for the components of the field of the surface wave (13), one can obtain the energy losses of a bunch over a unit path:

$$
\frac{d W^{s}}{d y}=\frac{\pi^{2}}{c^{2} \beta} \int_{0}^{\infty} d \omega \frac{\omega \exp \left\{-2 \frac{\omega}{v} a_{0}\right\}}{\left[1+\tilde{\kappa} \frac{\omega}{v}\right]^{2}}|\tilde{\eta}(\omega / v)|^{2} .
$$

Until now, we did not consider the influence of losses on the surface wave, which can be approximated for small $|\delta|$ if we use the first term $(\sim \delta)$ for the pole of the integrand in (9) (in the $k_{x}$ plane). One can show that if $|\delta| \ll 2(\beta+\kappa)$, the integrand in (13) should be multiplied by the factor 


$$
\exp \left\{-\frac{\delta^{\prime} \frac{|\omega x|}{v}-\delta^{\prime \prime}\left(|z|+a_{0}\right) \frac{\omega}{c}}{2\left(1+\tilde{\kappa} \frac{|\omega|}{v}\right)}\right\}
$$

where $\delta^{\prime}=\operatorname{Re} \delta$ and $\delta^{\prime \prime}=\operatorname{Im} \delta$. This expression determines the surface wave attenuation along the conductors. For example, if one suppose that the wires in Fig. 3 or in Fig. 4 are made of copper, then the typical scale of attenuation for the surface wave along the wires is no less than $1 \mathrm{~m}$.

\section{MOVEMENT THROUGH THE GRID}

In this problem, the charged-particle bunch moves perpendicularly to the grid with constant velocity $\vec{v}=v \overrightarrow{\mathbf{e}}_{z}$ [Fig. 2(b)]. As before, this bunch has infinitesimal size in the transverse directions (along the $x$ and $y$ axes) and some longitudinal distribution of charges $\eta(\zeta)$, where $\zeta=z-v t$. The charge density $\rho$ and the current density $\vec{j}$ now are given by the following expression:

$$
\rho=\delta(x) \delta(y) \eta(\zeta), \quad \vec{j}=v \rho \overrightarrow{\mathbf{e}}_{z}
$$

A similar problem has been previously considered in Ref. [7] with the point charge as a source. These results can be generalized for the case of the bunch (22) by substituting $q$ in Ref. [7] for $2 \pi \tilde{\eta}(\omega / v)$ in the corresponding formulas for the electric and magnetic fields or $q^{2}$ for $4 \pi|\tilde{\eta}(\omega / v)|^{2}$ in the corresponding formulas for the energy characteristics. Furthermore, we give only those expressions which are principal for the new conclusions concerning the properties of the radiation. Moreover, note that the structure of the surface waves was not deeply analyzed before.

As was described in Sec. II, we consider the total field as a sum of the incident one

$$
A_{z \omega}^{i}=\frac{1}{\pi c} \int_{-\infty}^{\infty} d k_{x} \int_{-\infty}^{\infty} d k_{y} \frac{\tilde{\eta}(\omega / v) e^{i \omega z / v+i k_{x} x+i k_{y} y}}{\frac{\omega^{2}}{c^{2}} \frac{1-\beta^{2}}{\beta^{2}}+k_{x}^{2}+k_{y}^{2}},
$$

and the field induced by the boundary

$$
\begin{aligned}
A_{x \omega}^{b}= & \frac{\omega}{\pi v} \int_{-\infty}^{\infty} d k_{x} \int_{-\infty}^{\infty} d k_{y}\left(\frac{\omega^{2}}{c^{2}} \frac{1-\beta^{2}}{\beta^{2}}+k_{x}^{2}+k_{y}^{2}\right)^{-1} \\
& \times \frac{k_{x} \exp \left\{i k_{x} x+i k_{y} y+i k_{z 0}|z|\right\} \tilde{\eta}(\omega / v)}{\left[\left(\frac{\omega^{2}}{c^{2}}-k_{x}^{2}\right)\left(1-i \tilde{\kappa} k_{z 0}\right)+\delta \frac{\omega}{c} k_{z 0}\right]}
\end{aligned}
$$

where $k_{z 0}=\sqrt{\frac{\omega^{2}}{c^{2}}-k_{x}^{2}-k_{y}^{2}}$ is fixed by the rules (4).

Using the saddle-point method for twofold integrals [12], one can calculate integral (24) in the far-field zone where $\omega / c \cdot R \gg 1\left(R=\sqrt{x^{2}+y^{2}+z^{2}}\right)$. The contribution of the saddle point represents the volume transient radiation, and the contribution of poles gives the surface wave.

\section{A. Surface wave}

If losses in wires are negligible $(\delta=0)$, the integrand in (24) has poles $k_{x}= \pm \omega / c$. The contribution of these poles represents the surface wave with the following nonzero components:

$$
\begin{aligned}
\left\{\begin{array}{c}
E_{y \omega}^{s} \\
E_{z \omega}^{s}
\end{array}\right\}= & \operatorname{sgn} x\left\{\begin{array}{c}
H_{z \omega}^{s} \\
-H_{y \omega}^{s}
\end{array}\right\}=\frac{-2 i \omega}{c^{2} \beta} \int_{0}^{\infty}\left\{\begin{array}{c}
\sin \left(k_{y} y\right) \\
\cos \left(k_{y} y\right) \operatorname{sgn} z
\end{array}\right\} \\
& \times \frac{k_{y} \exp \left\{-k_{y}|z|+i \frac{\omega}{c}|x|\right\} \tilde{\eta}(\omega / v)}{\left(1+\tilde{\kappa} k_{y}\right)\left(k_{y}^{2}+\omega^{2} v^{-2}\right)} d k_{y}
\end{aligned}
$$

Using (25), one can obtain expressions for the surface wave of a bunch with arbitrary longitudinal profile (3). In the case of a rectangular bunch (15), as an example, we have

$$
\begin{aligned}
\left\{\begin{array}{l}
E_{y}^{s} \\
E_{z}^{s}
\end{array}\right\}_{\text {rect }}= & -\frac{q \beta}{2 \sigma} \int_{0}^{\infty}\left\{\begin{array}{c}
\sin \left(k_{y} y\right) \\
\cos \left(k_{y} y\right) \operatorname{sgn} z
\end{array}\right\} \\
& \times \frac{e^{-k_{y} \hat{x}_{1}}-e^{-k_{y} \hat{x}_{2}}}{\left(1+\tilde{\kappa} k_{y}\right)} d k_{y},
\end{aligned}
$$

where $\hat{x}_{1,2}=|z|+|\beta(|x|-c t) \pm \sigma|$. The exact expression in terms of elementary functions can be obtained for a perfectly conducting plane in the $x$ direction $(\tilde{\kappa}=0)$ [11]:

$$
\left\{\begin{array}{l}
E_{y}^{s} \\
E_{z}^{s}
\end{array}\right\}_{\text {rect }}=-\frac{q \beta}{2 \sigma}\left\{\begin{array}{c}
\frac{y}{y^{2}+\hat{x}_{1}^{2}}-\frac{y}{y^{2}+\hat{x}_{2}^{2}} \\
\operatorname{sgn} z\left(\frac{\hat{x}_{1}}{y^{2}+\hat{x}_{1}^{2}}-\frac{\hat{x}_{2}}{y^{2}+\hat{x}_{2}^{2}}\right)
\end{array}\right\} .
$$

The expressions (25)-(27) show that the surface wave propagates along the wires at the speed of light in vacuum, like in the case in the previous section. Its shape does not change with time, and the wave does not decrease (if $\delta=0)$. The numerical results for the rectangular bunch are presented in Fig. 6, where both the field components and the energy flow density (18) are given. The pictures represent spatial distribution of the electric field and the energy flow of the surface wave in the plane of the wires (see Fig. 7 for reference). The distance between extrema (along the $x$ direction) of the field components corresponds to the length of the bunch.

One can calculate the total energy of the surface waves for bunches of various shapes by integrating the energy flux density over the planes $x= \pm x_{0}$, because the Poynting vector (18) of the surface wave is directed along the wires:

$$
\begin{aligned}
W^{s}= & \frac{16 \pi}{c^{3} \beta^{2}} \int_{0}^{\infty} d \omega \omega^{2}|\tilde{\eta}(\omega / v)|^{2} \\
& \times \int_{0}^{\infty} d k_{y} \frac{k_{y}}{\left(1+\tilde{\kappa} k_{y}\right)^{2}\left(k_{y}^{2}+\omega^{2} v^{-2}\right)^{2}} .
\end{aligned}
$$

This expression converges for any limited function $\tilde{\eta}(\omega / v)$. Thus, the total energy to generate a surface wave is limited 

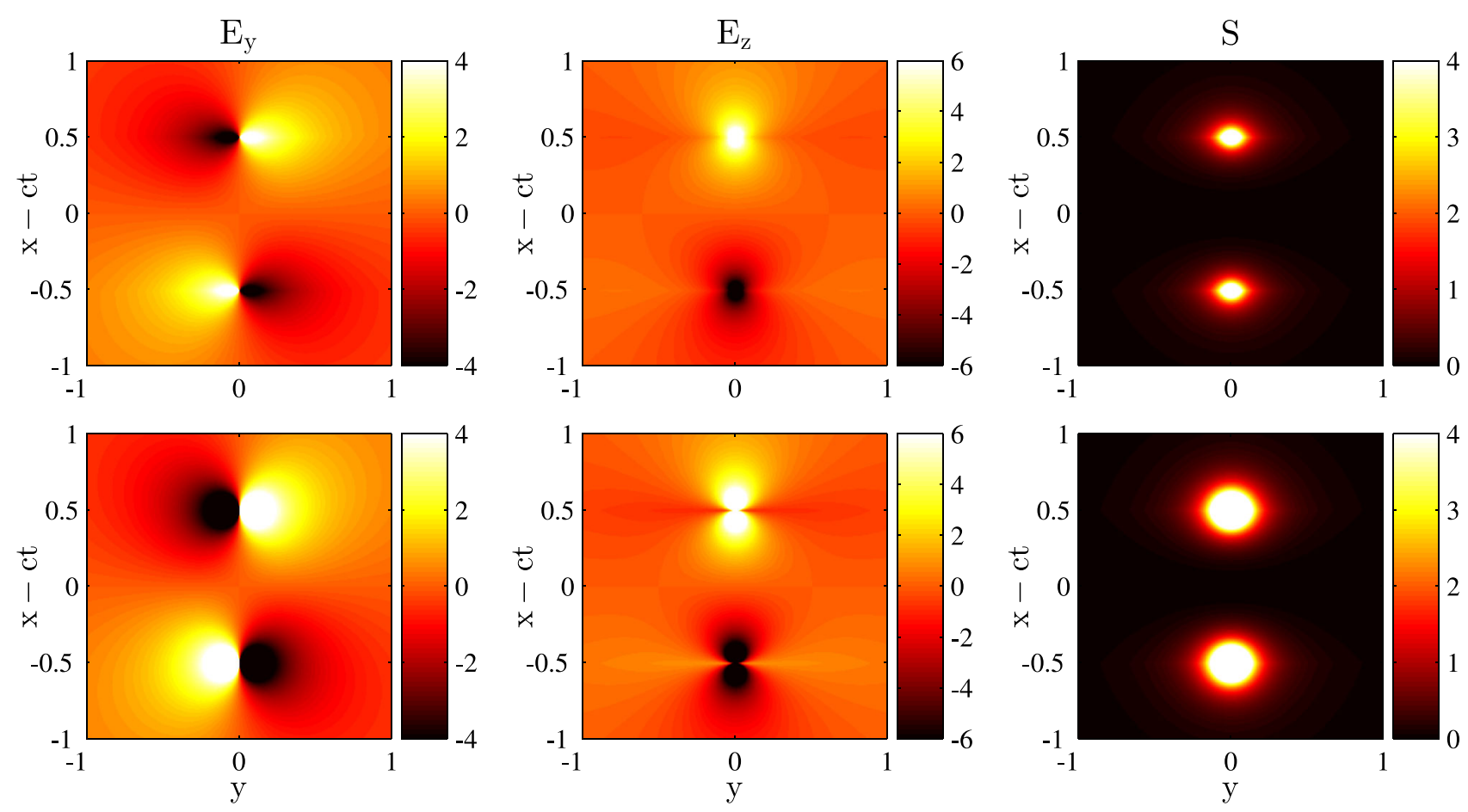

FIG. 6. The field components and the energy flow density of the surface wave from the rectangular bunch with $q=1$ esu. Gaussian units are used. The bunch length is $2 \sigma=1 \mathrm{~cm}$ for all pictures; $\tilde{\kappa}=0.0148 \mathrm{~cm}$ for the first row, and $\tilde{\kappa}=0$ for the second row; $\delta=0$, $z=+0, c t>\sigma$, and $\beta=1$.

for any bunches, including the point charge. This fact is true even for a plane perfectly conducting in the only direction where $\kappa=0$.

The influence of losses on the surface wave can be approximated for small $|\delta|$. One can show that if $|\delta| \ll 2(\beta+\kappa)$, the integrand in (25) should be multiplied by the factor

$$
\exp \left\{-\frac{\delta\left(\left|k_{y} x\right|+i|z| \omega / c\right)}{2\left(1+\tilde{\kappa}\left|k_{y}\right|\right)}\right\}
$$

Based on (29), one can estimate the maximum value of $E_{z \omega}^{s}$ at $z=0, y=0$ as follows:
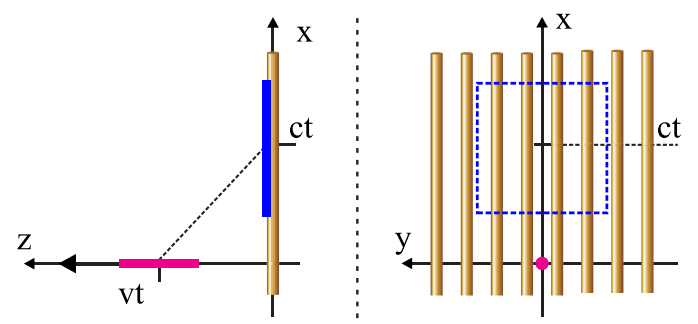

FIG. 7. Relative position of snapshots area, bunch, and wires for surface wave field distributions in Fig. 6. The area with calculated distributions is shown as a solid blue vertical line on the left projection and as a square with a dashed blue border on the right projection. The bold arrow shows the direction of the bunch motion.

$$
\begin{aligned}
\left.E_{z \omega}^{s}\right|_{y=z=0} \sim & \frac{-4 i q \omega}{\pi c^{2} \beta} \exp \left\{i \frac{\omega}{c}|x|\right\} \frac{\tilde{\kappa}^{2}}{(\operatorname{Re} \delta)^{2} x^{2}} \\
& \times\left[1-\left(1+\frac{|x| \operatorname{Re} \delta}{2 \tilde{\kappa}}\right) \exp \left\{-\frac{|x| \operatorname{Re} \delta}{2 \tilde{\kappa}}\right\}\right] .
\end{aligned}
$$

Far from the point where the charge intersects the grid, the value (30) inversely decreases to the second power of distance along the wires:

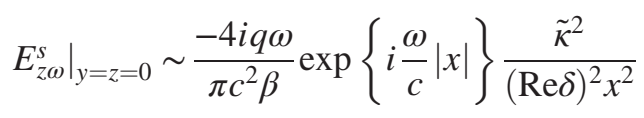

for $|x| \operatorname{Re} \delta /(2 \tilde{\kappa}) \gg 1$

\section{B. Volume radiation}

In the far-field zone, the field of volume radiation consists of two polarizations: $\left(E_{\theta}^{t r}, H_{\varphi}^{t r}\right)$ and $\left(E_{\varphi}^{t r}, H_{\theta}^{t r}\right)$. Some properties for both polarizations can be found in Ref. [7]. Furthermore, for convenience, we use expressions only for the total spectral density of volume radiation

$$
W_{\omega}^{t r}=\int_{0}^{\pi} d \theta \int_{0}^{2 \pi} d \varphi \sin \theta w_{\omega}^{t r}(\theta, \varphi),
$$

where $w_{\omega}^{t r}$ denotes the spectral angular density of radiated energy [7]: 

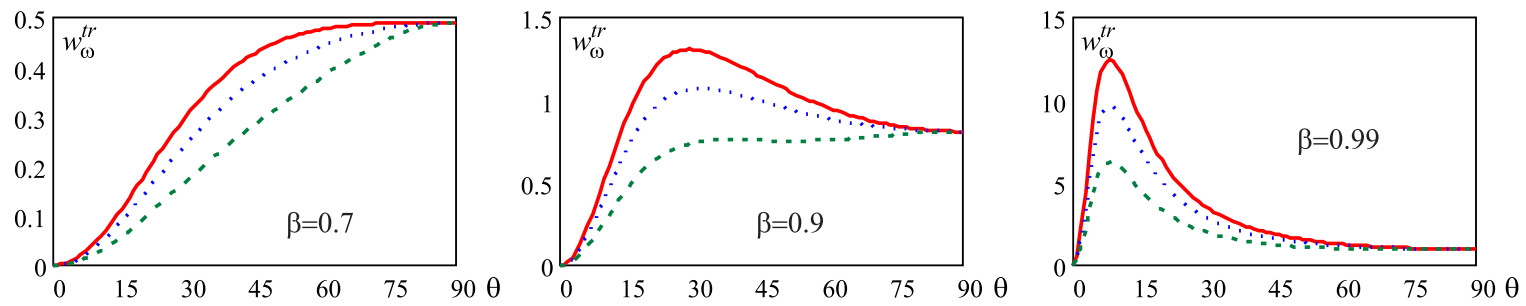

FIG. 8. Total angular spectral density of volume radiation $w_{\omega}^{t r}$ depending on $\theta$ for the case of perfectly conductive wires $(\delta=0)$. The energy density is measured in $4|\tilde{\eta}|^{2} / c$ units; the azimuthal angle is $\varphi=0 ; a=10 \mathrm{~mm} ; r_{0}=1 \mathrm{~mm}(\kappa \approx 0.09$, red solid line), $r_{0}=0.1 \mathrm{~mm}(\kappa \approx 0.55$, blue dotted line $)$, and $r_{0}=0.01 \mathrm{~mm}(\kappa \approx 1.01$, green dashed line $) ; \omega=\pi c /(5 a)$.

$$
w_{\omega}^{t r}=c R^{2}\left(\left|E_{\theta \omega}^{t r}\right|^{2}+\left|E_{\varphi \omega}^{t r}\right|^{2}\right) .
$$

The total spectral angular density of energy $w_{\omega}^{t r}$ is the sum of spectral angular densities for both polarizations (32) (due to their mutual orthogonality). Using expressions for the field of volume radiation, one can obtain

$$
\begin{aligned}
w_{\omega}^{t r}(\theta, \varphi)= & \frac{4 \beta^{2}}{c} \frac{\sin ^{2} \theta \cos ^{2} \theta \cos ^{2} \varphi}{\left(1-\sin ^{2} \theta \cos ^{2} \varphi\right)}\left|\tilde{\eta}\left(\frac{\omega}{c \beta}\right)\right|^{2} \\
& \times\left(1-\beta^{2} \cos ^{2} \theta\right)^{-2}|(1-i \kappa|\cos \theta|)+\delta| \cos \theta||^{-2} .
\end{aligned}
$$

The angular distributions (34) are symmetrical with respect to the $x y$ plane. In Figs. 8 and 9, the numerical results of the dependence of the total spectral energy density on $\theta$ and $\varphi$ for different charge velocities are represented.

For perfectly conducting wires ( $\delta=0$, Fig. 8$)$, the angles of maximum radiation can be analytically found. The azimuth of the maximum is always $\varphi_{\max }=0$ (in the transverse direction $\varphi=\pi / 2$, the volume radiation is absent). Analyzing the function $w_{\omega}^{t r}(\theta, 0)$, one obtains the following results.

If $\kappa^{2}<1 / 3$, then the function $w_{\omega}^{t r}(\theta, 0)$ has only one maximum: (i) for $\beta<\sqrt{\left(1+\kappa^{2}\right) / 2}$, the function $w_{\omega}^{t r}(\theta, 0)$ has a maximum at $\theta=\pi / 2$; (ii) for $\beta \geq \sqrt{\left(1+\kappa^{2}\right) / 2}$, the maximum is at

$$
\theta=\theta_{\max }(\beta, \kappa)=\operatorname{atan}\left(\sqrt{\frac{\beta\left(\kappa^{2}+1\right)-Q}{\beta\left(3 \kappa^{2}-1\right)+Q}}\right),
$$

where $Q=\sqrt{\left(\kappa^{2}+1\right)\left(\beta^{2}+9 \beta^{2} \kappa^{2}-8 \kappa^{2}\right)}$. The magnitude of $w_{\omega}^{t r}$ at $\theta=\pi / 2$ has a simple form:

$$
w_{\omega}^{t r}(\pi / 2,0)=\frac{4 \beta^{2}}{c}\left|\tilde{\eta}\left(\frac{\omega}{c \beta}\right)\right|^{2} .
$$

If $\kappa^{2}>1 / 3$, then three cases can be realized: (i) for $\beta \leq 4 \kappa / \sqrt{2+18 \kappa^{2}}$, the function $w_{\omega}^{t r}(\theta, 0)$ has only one maximum at $\theta=\pi / 2$; (ii) for $4 \kappa / \sqrt{2+18 \kappa^{2}}<\beta<$ $\sqrt{\left(1+\kappa^{2}\right) / 2}$, this function has two maximums at $\theta=$ $\pi / 2$ and $\theta=\theta_{\max }(\beta, \kappa)$ and a minimum at

$$
\theta=\theta_{\min }(\beta, \kappa)=\operatorname{atan}\left(\sqrt{\frac{\beta\left(\kappa^{2}+1\right)+Q}{\beta\left(3 \kappa^{2}-1\right)-Q}}\right)
$$

(iii) for $\beta>4 \kappa / \sqrt{2+18 \kappa^{2}}$, this function has only one maximum at $\theta=\theta_{\max }(\beta, \kappa)$, which is determined by Eq. (35).

For the "ideal grid," when both $\kappa=0$ and $\delta=0$, the spectral angular density of radiation in the plane $\varphi=0$ coincides with the corresponding value for the transition radiation of a point charge crossing the ideal metal plane [13]. The direction of the maximum of total volume radiation and its magnitude in this case are

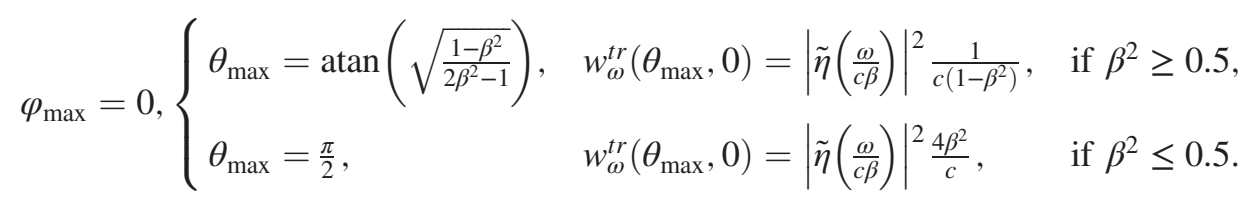

Figure 8 illustrates the angular spectral density of volume radiation for the grid of perfectly conducting wires. One can observe that the radiation energy in the direction of the wires $\left(\theta=90^{\circ}\right)$ does not depend on $\kappa$. The case of two radiation maximums can be observed in the second plot $(\beta=0.9$, dashed line). The difference between the maximums is notably small, so the radiation pattern is almost uniform for a wide range of angles.
The case of finite-conduction wires (copper) is shown in Fig. 9. The radiation in the direction of the wires $\left(\theta=90^{\circ}\right)$ is absent for real grids. As a rule, the pattern has the only maximum. However, the pattern width essentially depends on both the charge velocity and the wire thickness. In particular, notably wide patterns can be obtained (for example, in the case of $\beta=0.9$, $\varphi=0)$. 


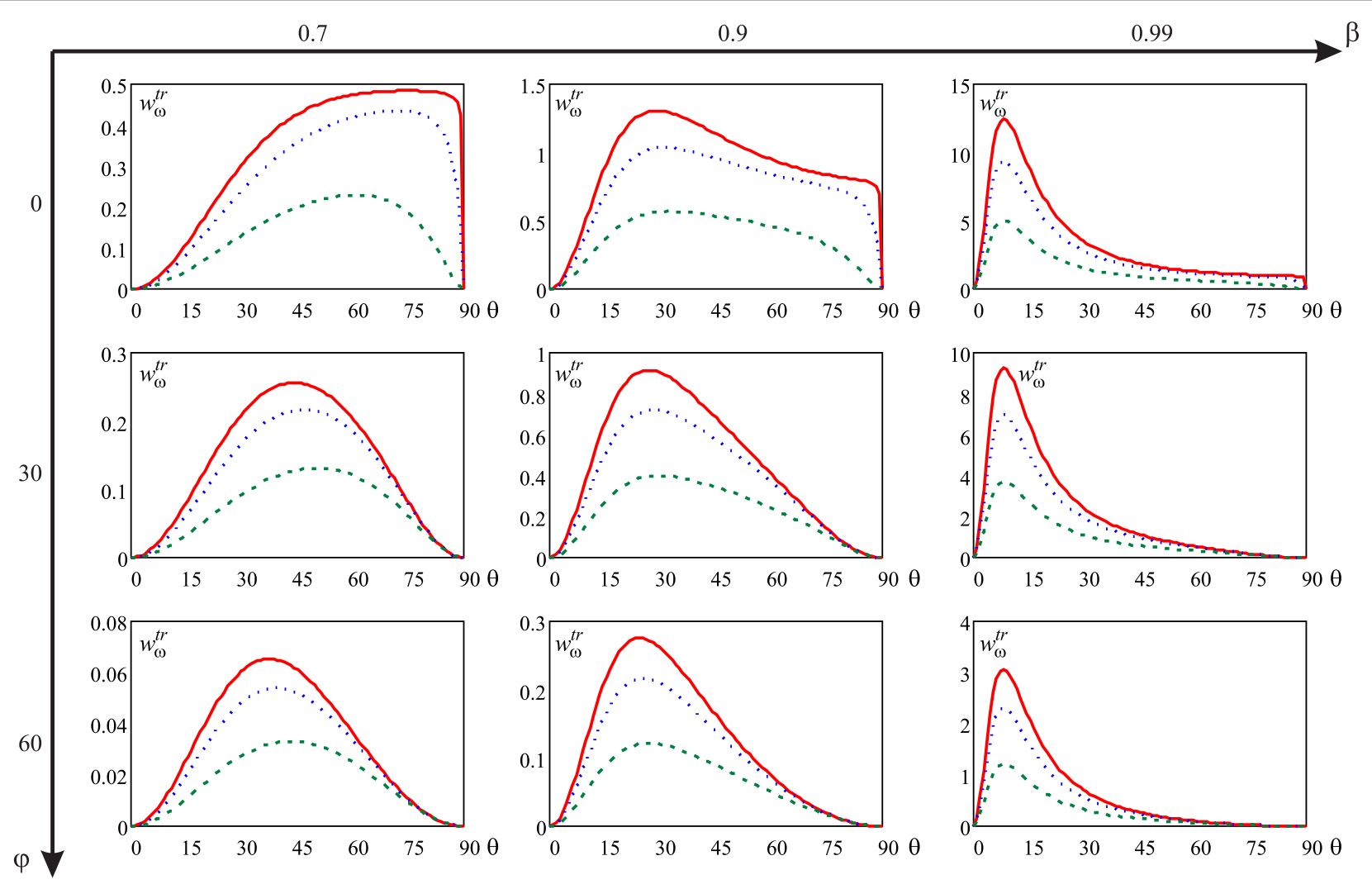

FIG. 9. Total angular spectral density of volume radiation $w_{\omega}^{t r}$ depending on $\theta$ for the case of copper wires. The energy density is measured in $4|\tilde{\eta}|^{2} / c$ units; $a=10 \mathrm{~mm} ; r_{0}=1 \mathrm{~mm}(\kappa \approx 0.09$, solid line $), r_{0}=0.1 \mathrm{~mm}(\kappa \approx 0.55$, dotted line $)$, and $r_{0}=0.01 \mathrm{~mm}$ $\left(\kappa \approx 1.01\right.$, dashed line); $\sigma_{e}=5.8 \times 10^{7} \mathrm{~S} / \mathrm{m} ; \omega=\pi c /(5 a)$. The azimuthal angle $\varphi$ and the charge velocity are shown in the external axes.

For a point charge (16), the spectral density $W_{\omega}^{\text {tr }}$ does not depend on frequency if $\kappa=0$, which indicates that the total energy of radiation is infinite. However, for charges with finite size, the total energy of the volume radiation $W^{t r}=$ $\int W_{\omega}^{t r} d \omega$ is always limited, because the integration is "limited" by the bunch profile's Fourier transform (15).

Note that the mentioned "energetic paradox" for a point charge can be easily solved if we consider $\kappa \neq 0$. For perfectly conductive wires $(\delta=0)$, one can integrate over $\omega, \varphi$, and $\theta$ and obtain the following simple result:

$$
\begin{aligned}
W_{\mathrm{pnt}}^{t r} & =\frac{q^{2} \beta}{2 \tilde{\kappa}} \ln \left(\frac{1+\beta}{1-\beta}\right) \\
& =\frac{\pi q^{2} \beta}{2 a}\left[\ln \left(\frac{a}{2 \pi r_{0}}\right)\right]^{-1} \ln \left(\frac{1+\beta}{1-\beta}\right) .
\end{aligned}
$$

Thus, the energy radiated from the point charge is limited for any charge velocity $\beta<1$ if we consider finite spacing of the structure $(a \neq 0)$.

\section{CONCLUSION}

We have investigated the radiation of charged-particle bunches that move parallel or perpendicularly to the plane grid composed of thin parallel wires by using the averaged boundary conditions method. In the approximation under consideration, the Smith-Purcell radiation is not taken into account. Therefore, in the case of parallel motion the charge generates only the surface waves, which propagate along the wires without divergence. The radiation losses over a unit path have been calculated. The form of the surface wave can be used to determine the sizes of bunches.

In the case of perpendicular motion, along with the surface wave the volume radiation occurs in the considered wavelength range. It is characterized by patterns that essentially depend on the structure parameters. In particular, both patterns with pronounced maxima and patterns with relatively small dependence in a wide range of angles can be obtained at certain parameters. The surface waves have a structure similar to the bunch profile, like in the case of parallel motion.

\section{ACKNOWLEDGMENTS}

This work was supported by grants of the President of Russian Federation (No. 273.2013.2) and Saint Petersburg State University (No. 11.0.61.2010). 
[1] V. V. Vorobev and A. V. Tyukhtin, Phys. Rev. Lett. 108, 184801 (2012).

[2] D. E. Fernandes, S. I. Maslovski, and M. G. Silveirinha, Phys. Rev. B 85, 155107 (2012).

[3] A. V. Tyukhtin and V. V. Vorobev, J. Opt. Soc. Am. B 30, 1524 (2013).

[4] A. V. Tyukhtin and V. V. Vorobev, Phys. Rev. E 89, 013202 (2014).

[5] K. A. Barsukov and L. G. Naryshkina, Sov. Radiophys. 8, 667 (1965).

[6] K. A. Barsukov and L. G. Naryshkina, Radiophys. Quantum Electron. 10, 276 (1967).

[7] V. N. Krasil'nikov and A. V. Tyukhtin, Radiophys. Quantum Electron. 33, 945 (1990).

[8] B. M. Bolotovskii and A. V. Serov, J. Exp. Theor. Phys. 98, 1109 (2004).
[9] C. F. Papadopoulos, R. Fiorito, R. Kishek, P. O'Shea, A. Shkvarunets, M. Conde, W. Gai, and J. Power, in Proceedings of the 23rd Particle Accelerator Conference, Vancouver, Canada, 2009 (IEEE, Piscataway, NJ, 2009), pp. 4072-4074.

[10] M. I. Kontorovich, M. I. Astrakhan, V. P. Akimov, and G. A. Fersman, Electrodynamics of Grid Structures (Radio Svyaz, Moscow, 1987) [in Russian].

[11] A. P. Prudnikov, Y. A. Brychkov, and O. I. Marichev, Integrals and Series. Elementary Functions (Nauka, Moscow, 1981).

[12] L. B. Felsen and N. Marcuwitz, Radiation and Scattering of Waves (Prentice-Hall, Englewood Cliffs, NJ, 1973).

[13] V. L. Ginzburg and V. N. Tsytovich, Transition Radiation and Transition Scattering (Adam Hilger, London, 1990). 